\title{
Reconhecimento de dígitos aplicado à automação de ensaios em equipamentos médicos
}

\author{
Josemir da Cruz Alexandrino*, Alexandre Henrique Hermini, Herman Augusto Lepikson, \\ Handerson Jorge Dourado Leite, Ricardo de Araújo Kalid
}

Resumo Apresenta-se o desenvolvimento de um aplicativo para a captura da imagem de displays numéricos, reconhecimento dos dígitos nela contidos e envio dos valores reconhecidos para um sistema que permita gerenciar ensaios de desempenho metrológico em diversos tipos de equipamentos médicos. Utilizando uma webcam e a ferramenta de desenvolvimento Delphi ${ }^{\circledR}$, foi criado um algoritmo baseado na segmentação dos dígitos e contagem dos pontos contidos em cada segmento. Tal contagem resulta em um conjunto numérico, como uma espécie de assinatura do dígito. O software possui as fases de treinamento e reconhecimento, além de um modo de avaliação. Para avaliar o desempenho do aplicativo foram aplicados três testes. No primeiro, dez mil valores diferentes foram gerados e reconhecidos, utilizando três webcams de marcas diferentes. No segundo, usando três equipamentos diferentes, foi realizado o reconhecimento de 100 leituras diferentes e a taxa de acerto verificada por comparação visual. No terceiro, o aplicativo foi ajustado para o reconhecimento da temperatura indicada no display de uma incubadora neonatal e comandado, através da interface de comunicação entre aplicativos, por uma versão protótipo de um sistema de gerenciamento de ensaios em equipamentos médicos. Os resultados do primeiro teste apresentaram 100\% de acertos para duas webcams e $99,96 \%$ para uma terceira. Nos outros testes a taxa de acerto do aplicativo foi de $100 \%$, demonstrando que a aplicação é factível e segura.

Palavras-chave Reconhecimento de caracteres, Avaliação de equipamentos médicos, Metrologia, Engenharia clínica.

\section{Digit recognition applied to the automation of evaluation of medical equipment}

\begin{abstract}
This paper presents the development of an application for capturing an image of numeric displays, recognizing the digits contained therein and transferring them to an automation system that manages metrological performance tests on different types of medical equipment. Using a webcam and the Delphi $i^{\circledR}$ development tool, an algorithm was created based on segmentation of the digits and counting of the points contained in each segment. This counting results in a numerical set, a kind of signature of the digit. The software has the phases of training and recognition, as well as an evaluation mode. Three tests were applied to evaluate the performance of the application. At first, ten thousand different values were generated and recognized using three webcams of different manufacturers. In the second, using three different equipments, there was the recognition of 100 different readings and the hit rate verified by visual comparison. In the third, the application was set for recognizing the temperature indicated on the display of a neonatal incubator and controlled through the communication interface between applications, by a prototype version of a management system of medical equipment assays. Tests conducted in the first way resulted in a hit rate of $100 \%$ for two webcams and $99.96 \%$ for a third. In other tests the hit rate was $100 \%$, demonstrating that the application is feasible and safe.
\end{abstract}

Keywords Character recognition, Evaluation of medical equipment, Metrology, Clinical engineering. 


\section{Introdução}

Dentre os componentes dos serviços de saúde, os equipamentos médicos são os que mais têm contribuído para a melhoria do diagnóstico e da terapêutica de várias doenças (Figueiredo, 2008). Possuem, entretanto, fatores de risco inerentes, podendo estar relacionados a vários tipos de erros (Garg et al., 2002; Kaczmarek et al., 2000), inclusive devido à calibração inexistente ou inadequada (Fitzgibbon et al., 2004).

No Brasil, ensaios de desempenho metrológico em equipamentos médicos são exigidos apenas na fase de pré-comercialização (Brasil, 2007). Durante a fase de utilização dos equipamentos pouco se tem feito, possivelmente devido à falta de recursos humanos especializados e de uma cultura de manutenção inadequada. Isto resulta na falta de monitoração e controle dos fatores de riscos associados ao desempenho e segurança dos equipamentos em uso. Evidências de que essas preocupações podem ser verdadeiras são encontradas nos estudos realizados por Leite et al. (2004) e Miranda et al. (2002).

Recursos humanos, método, instrumentos padrões para a medição das variáveis e recursos auxiliares são componentes essenciais para a execução de ensaios de avaliação do desempenho de um equipamento. Nos ensaios pouco automatizados a maior dependência é do recurso humano. O método ajuda a minimizar esta dependência se é seguido integralmente. Com a automatização do sistema é possível diminuir a dependência humana, aumentar a confiabilidade e reduzir o tempo de execução. Entretanto, muitos equipamentos médicos não possuem interface de comunicação que transmita para um computador os valores das variáveis medidas ou controladas. Isso dificulta a automatização do sistema.

Embora diversos sistemas para a automatização deste processo sejam encontrados na literatura especializada, poucos tratam a questão da conectividade com equipamentos sem interface de comunicação própria. Alegria e Serra (2000) desenvolveram um sistema para a captura de imagens dos displays dos equipamentos e reconhecimento dos seus dígitos. $\mathrm{O}$ sistema utiliza um algoritmo que divide cada dígito em 15 regiões retangulares, determina quais regiões estão "acesas" e, com o auxílio de uma tabela, obtém os valores dos dígitos. Foiatto e Roehe (2006), e posteriormente Lima et al. (2008), apresentaram sistemas semelhantes, utilizando uma máscara de verificação dos segmentos, posicionada manualmente, para reconhecimento de displays de sete segmentos.

De um modo mais amplo, o problema de reconhecer caracteres desconectados, não manuscritos e estáticos é considerado bem resolvido. Porém, na aplicação desejada, reconhecimento de valores indicados em displays de sete segmentos ou matriciais, características de ordem prática, como a facilidade de implementação e o custo computacional compatível com uma utilização em tempo real (mínimo de duas leituras por segundo), são condições que devem ser consideradas. Dessa forma, apresenta-se o desenvolvimento de um aplicativo para a captura da imagem de um display, reconhecimento dos dígitos nela contidos e envio dos valores reconhecidos para um sistema de automação que permita gerenciar ensaios de desempenho metrológico em diversos tipos de equipamentos médicos.

\section{Materiais e Métodos}

O projeto foi estruturado em quatro partes: 1) Captura e pré-processamento das imagens provenientes da webcam, 2) Interface com o usuário, 3) Lógica de delimitação e contagem dos dígitos e, 4) Lógica dos modos de treinamento, reconhecimento e avaliação.

A captura das imagens foi implementada com componentes do DSPack (Gourvest et al., 2010), uma biblioteca de software livre para aplicações multimídias. Um componente denominado VideoWindow, visível ao usuário, gerencia e exibe a reprodução do vídeo. Em intervalos regulares ou a um clique do mouse, um quadro é obtido e convertido em uma imagem em preto e branco, conforme as seguintes condições:

$$
\left\{\begin{array}{l}
P_{\mathrm{x}, \mathrm{y}}=0 \text { para }\left(0,2989 R_{\mathrm{x}, \mathrm{y}}+0,587 G_{\mathrm{x}, \mathrm{y}}+0,114 B_{\mathrm{x}, \mathrm{y}}\right) \\
V R<\text { Threshold } V R \\
P_{\mathrm{x}, \mathrm{y}}=1 \text { caso contrário }
\end{array}\right.
$$

O termo entre parênteses expressa a luminância monocromática do ponto de coordenadas $(x, y)$, correspondendo a um valor em uma escala de tons de cinza (Plataniotis e Venetsanopoulos, 2000). O Threshold é o limite sobre o qual o ponto é binarizado (Sahoo et al., 1988). VR vale-1 para imagens em vídeo reverso (dígitos claros sobre fundo escuro) ou 1 para imagens normais (dígitos escuros sobre fundo claro). O ajuste do Threshold pode ser realizado pelo usuário ou determinado como o ponto médio entre os dois máximos do histograma da imagem em tons de cinza.

A interface com o usuário foi concebida para que fosse possível selecionar dispositivos, particularmente a webcam, executar funções acessórias e definir parâmetros usados nas lógicas e modos de funcionamento. Possibilita também a apresentação das imagens, dos itens selecionados, dos parâmetros ajustados, dos resultados de treinamento, reconhecimento ou avaliação e dos valores usados na simulação de um display para fins de avaliação. Outro aspecto que completa a interface do aplicativo com o 
mundo externo é a capacidade de se comunicar com outro aplicativo para receber comandos e informar os valores reconhecidos. Neste caso foi utilizado o mecanismo de mensagens do Windows ${ }^{\circledR}$.

Na lógica de delimitação e contagem, o usuário marca com o mouse sobre a imagem um retângulo contendo os dígitos, e um procedimento de varredura encontra as coordenadas e a quantidade de cada dígito. Alternativamente, foi desenvolvido um algoritmo de busca automática que utiliza o histograma das linhas da imagem para determinar a região horizontal compatível com a altura dos dígitos e, de forma análoga, o histograma das colunas desta região, visando localizar regiões menores, compatíveis com a largura dos dígitos.

Os modos de treinamento e reconhecimento são baseados na obtenção de um conjunto numérico próprio de cada dígito (assinatura) a partir de um mecanismo de segmentação (Figura 1). Tal mecanismo consiste em dividir a imagem de cada dígito em segmentos e contar o número de pontos pretos contidos em cada segmento. Por simplificação, optou-se por uma segmentação matricial de duas colunas por um número variável de linhas (Segments), definido pelo usuário.

Enquanto o modo de treinamento visa obter um conjunto padrão formado pelas assinaturas dos dígitos de 0 a 9, o modo de reconhecimento objetiva reconhecer os dígitos do display através da obtenção das suas assinaturas e da comparação destas com as assinaturas padrão. Várias formas de avaliar a semelhança entre as assinaturas foram testadas. A que se mostrou mais apropriada foi aquela em que, para cada dígito do padrão, era calculada a soma das diferenças absolutas entre os elementos das assinaturas do dígito a reconhecer e do padrão. Em seguida, o dígito é reconhecido pela menor soma. Neste caso, uma margem de tolerância, definida experimentalmente em $30 \%$ da soma dos elementos do dígito a reconhecer evita que qualquer símbolo possa ser reconhecido como um dígito.

Um modo de avaliação foi adicionado ao aplicativo para disponibilizar um método automático de verificação da taxa de acerto dos reconhecimentos para um número massivo de amostras. Neste modo, o aplicativo simula um display com valores previamente

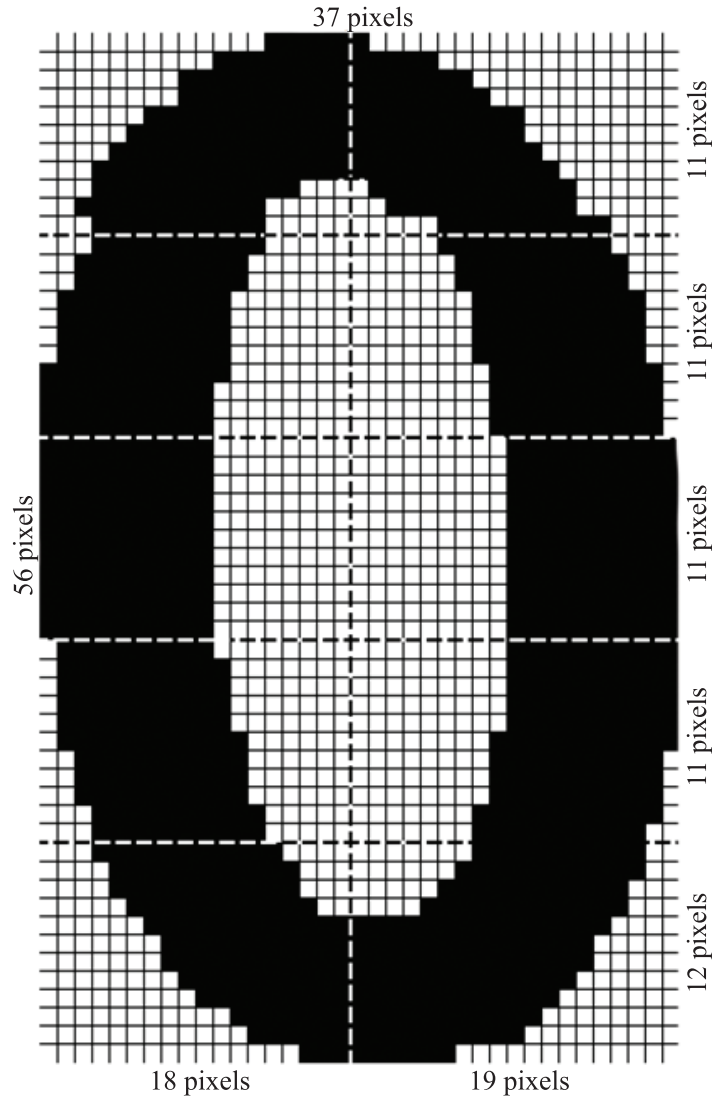

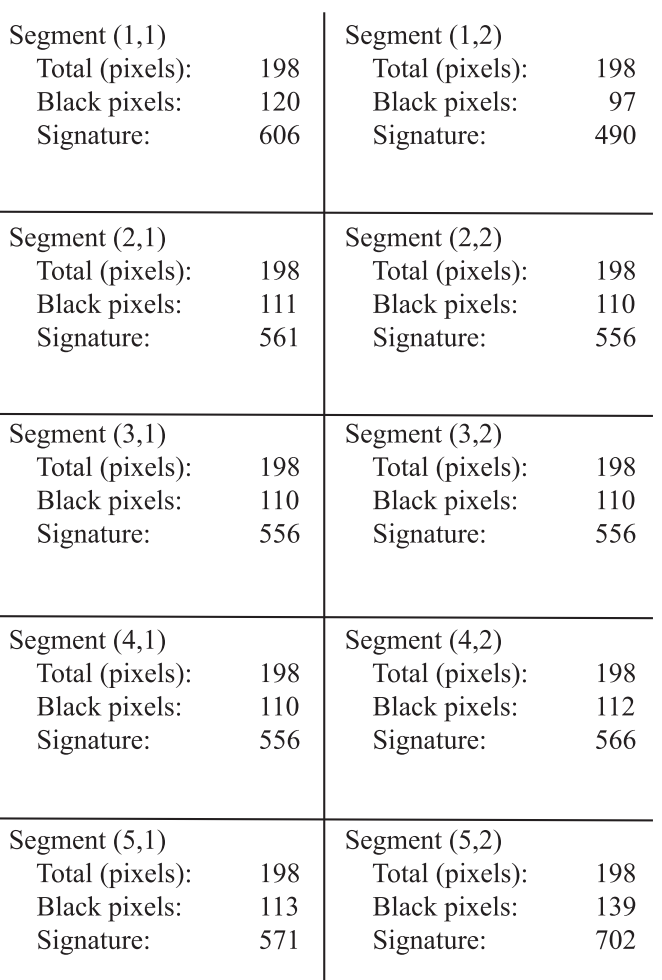

Signature $=1000 *$ blackpixels DIV totalpixels

Figura 1. Detalhe da segmentação e obtenção da assinatura do dígito 0 .

Figure 1. Detail of the segmentation and capture of the signature of the digit zero. 
conhecidos e a webcam é apontada para esse display. Desta forma, comparando o valor gerado com o reconhecido, é possível estabelecer automaticamente a taxa de acerto, que é apresentada ao lado de cada valor reconhecido.

Para avaliar o desempenho do aplicativo foram utilizados três tipos de teste. No primeiro, o aplicativo foi colocado no modo de avaliação e dez mil valores diferentes $(00,00$ a 99,99$)$ foram gerados e reconhecidos. Três webcams de marcas diferentes foram utilizadas para este teste.

No segundo teste, o aplicativo foi submetido ao reconhecimento de 100 leituras diferentes e a taxa de acerto foi verificada pelo usuário, por comparação visual. Três equipamentos diferentes, com displays de formatos diferentes foram usados no segundo teste. No terceiro, o aplicativo foi ajustado para o reconhecimento da temperatura indicada no display de uma incubadora neonatal e comandado, através da interface de comunicação entre aplicativos, por uma versão do protótipo de um sistema de gerenciamento de ensaios em equipamentos médicos.

No último teste, o sistema foi configurado para se conectar com o protótipo de um analisador de incubadoras, se comunicar com o aplicativo de reconhecimento de dígitos e solicitar os valores reconhecidos durante a execução do ensaio de verificação do erro da temperatura indicada pela incubadora. $\mathrm{O}$ ensaio foi executado para as temperaturas de controle (ajustada na incubadora) de 32 e $36{ }^{\circ} \mathrm{C}$.

$\mathrm{O}$ aplicativo e o sistema foram instalados no Laboratório de Engenharia Clínica do Instituto Federal da Bahia (onde os produtos foram desenvolvidos), em um computador com processador Pentium D de 2,8 GHz, 480 MB de RAM, sistema operacional Windows ${ }^{\circledR}$ XP com Service Pack 2 e monitor Samsung 793v, ajustado com resolução de $1024 \times 768$ pixels. O parâmetro Segments foi ajustado para $5(5 \times 2=10$ segmentos $)$ e a webcam colocada a uma distância de $18 \mathrm{~cm}$, com o seu eixo focal perpendicular à superfície dos displays. Os parâmetros de brilho e contraste do filtro gráfico da webcam foram ajustados de forma a se obter uma imagem com os contornos dos dígitos bem definidos. O número de segmentos foi determinado de forma experimental, iniciando em $1 \times 2$ e sendo gradativamente aumentado até se verificar a convergência da taxa de acerto.

\section{Resultados}

As condições e resultados dos testes de avaliação do aplicativo encontram-se sumarizados na Tabela 1. No teste do tipo 1, com as duas primeiras webcams, obteve-se uma taxa de acerto de $100 \%$, isto é, dez mil reconhecimentos corretos, e 99,96\% com a terceira webcam. Os testes foram repetidos três vezes, entretanto as taxas de acerto mantiveram-se inalteradas. Foi observada uma velocidade máxima de cinco reconhecimentos por segundo. No teste do tipo 2, foram utilizados um multímetro com display de LCD de sete segmentos sem backlight, um bisturi elétrico com display de LEDs, e uma incubadora com display de LCD, do tipo matriz ativa. Em todos os casos foram obtidas taxas de $100 \%$ de acertos. No teste tipo 3, o sistema colheu 1.828 leituras do aplicativo, durante as duas repetições do ensaio (nas temperaturas de controle de 32 e $36{ }^{\circ} \mathrm{C}$ ), mas apenas dois lotes de 720 leituras, correspondentes aos intervalos de temperatura estável, foram processados. A Figura 2 apresenta o gráfico com estes valores, como também os valores medidos com o analisador (padrão). Os resultados do ensaio são apresentados na Tabela 2.

\section{Discussão}

Um problema encontrado na captação das imagens do display de LCD foi atribuído ao elevado tempo de resposta, típico desses dispositivos. Algumas vezes a imagem era obtida no momento que um dígito estava mudando de valor. A imagem então registrava os dois valores sobrepostos. Isso fazia com que a lógica de reconhecimento respondesse com um código de erro, pois a assinatura obtida não se assemelhava a nenhuma do padrão. Isso foi solucionado fazendo com

Tabela 1. Resultados da avaliação do aplicativo.

Table 1. Assessment results of application.

\begin{tabular}{|c|c|c|c|c|c|c|}
\hline Tipo do teste & Webcam & $\begin{array}{l}\text { Número de } \\
\text { leituras }\end{array}$ & $\begin{array}{l}\text { Número de } \\
\text { repetições }\end{array}$ & Equipamento & $\begin{array}{l}\text { Tipo de } \\
\text { display }\end{array}$ & $\begin{array}{c}\text { Taxa de } \\
\text { acerto }(\%)\end{array}$ \\
\hline \multirow{3}{*}{1} & 1 & \multirow{3}{*}{10.000} & 3 & \multirow{3}{*}{$\begin{array}{l}\text { Monitor de } \\
\text { computador }\end{array}$} & \multirow{3}{*}{ TRC } & 100,00 \\
\hline & 2 & & 3 & & & 100,00 \\
\hline & 3 & & 3 & & & 99,96 \\
\hline \multirow{3}{*}{2} & \multirow{4}{*}{1} & 100 & 3 & Multímetro & LCD & 100,00 \\
\hline & & 100 & 3 & Bisturi AF & LED & 100,00 \\
\hline & & 100 & 3 & \multirow{2}{*}{ Incubadora } & \multirow{2}{*}{ Matriz } & 100,00 \\
\hline 3 & & 720 & 2 & & & 100,00 \\
\hline
\end{tabular}




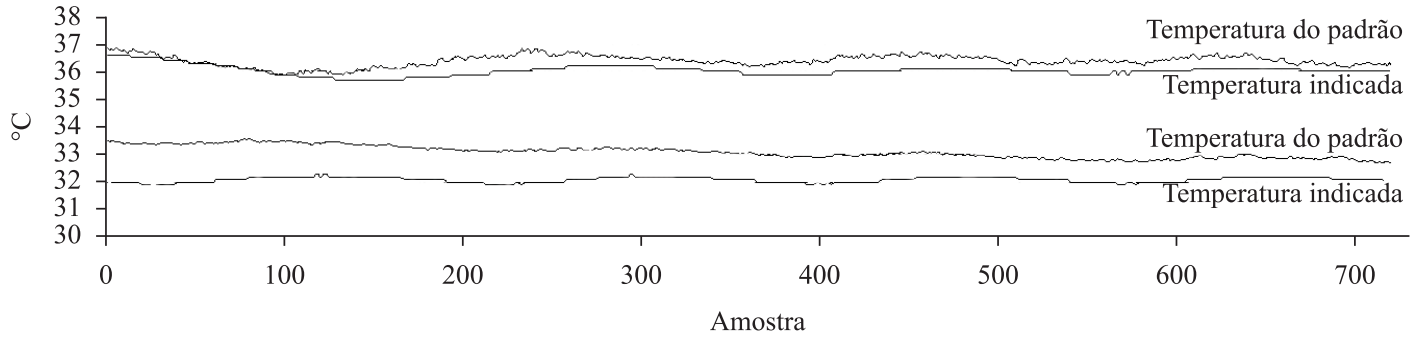

Figura 2. Temperaturas indicadas pela incubadora (obtidas pelo aplicativo) e medidas com o padrão.

Figure 2. Temperatures indicated by the incubator (obtained by the application) and standard temperatures.

Tabela 2. Resultados do ensaio do erro da temperatura indicada pela incubadora.

Table 2. Assay results of the error of the temperature indicated by the incubator.

\begin{tabular}{cccccc}
\hline \multicolumn{2}{c}{ Temperatura média } & Erro & $\begin{array}{c}\text { Valor de } \\
\text { referência }\end{array}$ & Conformidade & $\begin{array}{c}\text { Item normativo } \\
\text { de referência }\end{array}$ \\
\hline 36,04 & 36,39 & $-0,35$ & 0,8 (máximo) & Conforme & NBR IEC 60.601-2-19/50.106 \\
32,09 & 33,09 & $-1,00$ & 0,8 (máximo) & Não conforme & NBR IEC 60.601-2-19/50.106 \\
\hline
\end{tabular}

Obs.: valores expressos $\mathrm{em}{ }^{\circ} \mathrm{C}$.

que todo o processo de reconhecimento, inclusive a obtenção de um novo quadro da imagem, fosse repetido em um loop, por um número máximo de vezes, atualmente ajustado em 20.

Com relação aos testes do tipo 1 , observou-se bons resultados. No pior caso, a taxa de $99,96 \%$ de acertos correspondeu a 4 erros em 10 mil leituras. Entretanto, com uma pequena modificação no aplicativo, foi possível detectar que esse tipo de falha estava relacionado à etapa de captação das imagens. Como esse tipo de erro só ocorreu com uma marca de webcam, inferiu-se que o problema estava neste dispositivo. De qualquer forma, testes mais apurados devem ser realizados para um diagnóstico mais preciso. A velocidade máxima de cinco leituras por segundo foi outro resultado interessante, considerando uma taxa de atualização de 0,5 segundo, típica dos displays digitais dos equipamentos.

Também foi constatada a boa adequação do sistema de reconhecimento de dígitos a diferentes tipos de displays (100\% de acertos nos testes do tipo 2$)$, característica importante devido à grande diversidade de equipamentos médicos encontrados no mercado. Entretanto, o teste com o multímetro apresentou forte dependência da luminosidade ambiente, pois o seu display não possuía fonte de luz própria.

Com os teste do tipo 3, foi possível avaliar todas as funcionalidades do aplicativo e comprovar os reais benefícios da solução, ou seja, eliminação de erros grosseiros na leitura, registros corretos dos valores indicados, liberação do ser humano para a execução de tarefas mais nobres, excluindo-se tarefas enfadonhas, possibilidade de efetuar um maior número de leituras e a consequente melhoria da qualidade metrológica dos ensaios, entre outros. Agrega-se ainda o benefício de uma solução de baixo custo.

Por fim, é importante ressaltar que os ajustes dos parâmetros de brilho e de contraste do filtro gráfico da webcam foram fundamentais para o bom funcionamento do aplicativo e tiveram que ser ajustados de forma a se obter uma imagem com os contornos dos dígitos bem definidos.

\section{Conclusão}

Foi apresentado o desenvolvimento de um aplicativo para captura de imagens e reconhecimento dos dígitos numéricos apresentados em displays, visando à automação de ensaios de avaliação metrológica em equipamentos médicos. Todos os testes executados e seus resultados corroboram as necessidades preestabelecidas com um nível adequado de confiança.

$\mathrm{O}$ ajuste do brilho e do contraste da imagem, o posicionamento da câmara e, em alguns casos, a luminosidade do ambiente foram fatores críticos para o bom funcionamento do aplicativo, pois afetaram a qualidade da imagem. Dessa forma, especial atenção deve ser dada ao ajuste destes fatores antes de se iniciar a operação do aplicativo.

Um possível desenvolvimento do aplicativo para mostradores analógicos pode ampliar o escopo de equipamentos médicos acopláveis ao sistema de avaliação de desempenho e permitir o seu emprego como parte de outros sistemas de calibração que extrapolem a área da engenharia clínica. 


\section{Referências}

Alegria FC, Serra AC. Computer vision applied to the automatic calibration of measuring instruments. Measurement. 2000; 28:185-95. http://dx.doi.org/10.1016/ S0263-2241(00)00011-7

Brasil. Agência Nacional de Vigilância Sanitária. Resolução $\mathrm{RDC}^{\circ}$ 32, de 29 de maio de 2007. Dispõe sobre a certificação compulsória dos equipamentos elétricos sob regime de Vigilância Sanitária e dá outras providências. Diário Oficial da República Federativa do Brasil, Poder Executivo, Brasília, DF, 01 jun 2007.

Figueiredo JAF. Inovação e desempenho de equipamentos médicos - estudo qualitativo em hospitais portugueses [tese]. Minho: Universidade de Minho; 2008.

Fitzgibbon DR, Posner KL, Domino KB, Capplan RA, Lee LA, Cheney FW. Chronic pain management: American Society of Anesthesiologists Closed Claims Project. Anesthesiology. 2004; 100(1): 98-105. PMid:14695730. http://dx.doi.org/10.1097/00000542-200401000-00018

Foiatto N, Roehe JML. Sistema automatizado de calibração para medidores digitais a partir da captura de imagens e interface de comunicação GPIB (IEEE 488). In: Congresso e Feira de Qualidade em Metrologia - ENQUALAB: Anais do Congresso e Feira de Qualidade em Metrologia; 2006 mai 30-01; São Paulo, Brasil. São Paulo: REMESP; 2006.

Garg A, Wadhwa M, Brown K, Luckett C, Vaughn T, Birgersdotter-Green U, Feld G. Inappropriate implantable cardioverter defibrillator discharge from sensing of external alternating current leak. Journal of Interventional Cardiac Electrophysiology. 2002; 7(2): 181-4. PMid:12397229. http://dx.doi.org/10.1023/A:1020846007149

Gourvest H, Nevhasymyy A, Offenwanger M, Mitrovic M, Steffensen M. The DSPack Project [Internet]. 2010 [cited 2010 Jan 25]. Available from: http://www.progdigy. com/?page_id=4

Kaczmarek RG, Beaulieu MD, Kessler LG. Medical device tracking: results of a case study of the implantable cardioverter defibrillator. American Journal of Cardiology. 2000; 85(5): 588-92. http://dx.doi.org/10.1016/S00029149(99)00816-4

Leite HJD, Silva HAN, Alexandrino JC. Esfigmomanômetros: quais as condições após sete anos de publicação do regulamento metrológico? In: Congresso Latino Americano de Engenharia Biomédica- IFMBE: Anais do III Congresso Latino Americano de Engenharia Biomédica; 2004 set 22-25; João Pessoa, Brasil. IFMBE; 2004. V. 5, n. 1, p. 983-6.

Lima DA, Pereira GAS, Vasconcelos FH. A computer vision system to read meter displays. In: IMEKO Symposium: Proceedings of the $16^{\text {th }}$ IMEKO Symposium [Internet]; 2008; Florence. [cited: $2010 \mathrm{Jul}$ 9]. Available from: http://www. imeko.org/publications/tc4-2008/imeko-tc4-2008-122.pdf

Miranda ICS, Pedroso JCL, Amaral JLG, Brito LC, Calil SJ Electrical safety performance assessment of medical electrical equipment used in the practice of anesthesia. In: Raising the Profile of Clinical Engineering: Proceedings of the Raising the Profile of Clinical Engineering; 2002 May 17; Dublin, Ireland. York. Institute of Physics and Engineering in Medicine; 2002. p. 22.

Plataniotis KN, Venetsanopoulos AN. Color Image Processing and Applications (Digital Image Processing) [Internet]. Berlin: Springer; 2000 [acess 2011 Aug 05]. Available from: http://www.dsp.utoronto.ca/ kostas/Publications2008/pub/ bookchapters/2000-SpringerMonograph.pdf

Sahoo PK, Soltani S, Wong AKC. A survey of thresholding techniques. Computer Vision, Graphics, and Image Processing. 1988; 41(2): 233-60. http://dx.doi.org/10.1016/0734$189 X(88) 90022-9$

\footnotetext{
Autores

Josemir da Cruz Alexandrino

Programa de Pós-graduação em Engenharia Industrial, Universidade Federal da Bahia - UFBA, Rua Professor Aristides Novis, 02, Federação, CEP 40210-630, Salvador, BA, Brasil

Núcleo de Tecnologia em Saúde, Instituto Federal de Educação, Ciência e Tecnologia da Bahia - IFBA,

Rua Emídio dos Santos, s/n, Barbalho, CEP 40301-015, Salvador, BA, Brasil
}

\section{Alexandre Henrique Hermini}

Centro de Atenção Integral à Saúde da Mulher, Universidade de Campinas - UNICAMP,

Av. Alexander Fleming, 101, Cidade Universitária “Zeferino Vaz”, Barão Geraldo, CEP 13083-881, Campinas, SP, Brasil

\section{Herman Augusto Lepikson, Ricardo de Araújo Kalid}

Programa de Pós-graduação em Engenharia Industrial, Universidade Federal da Bahia - UFBA,

Rua Professor Aristides Novis, 02, Federação, CEP 40210-630, Salvador, BA, Brasil

\section{Handerson Jorge Dourado Leite}

Núcleo de Tecnologia em Saúde, Instituto Federal de Educação, Ciência e Tecnologia da Bahia - IFBA,

Rua Emídio dos Santos, s/n, Barbalho, CEP 40301-015, Salvador, BA, Brasil 